\title{
Éditer un cahier de travail de Montesquieu
}

Les apports du numérique et de la TEI

\section{Pierre-Yves Buard et Carole Dornier}

\section{(2) OpenEdition}

\section{Journals}

Édition électronique

URL : http://journals.openedition.org/recherchestravaux/100

DOI : $10.4000 /$ recherchestravaux. 100

ISSN : 1969-6434

Éditeur

UGA Éditions/Université Grenoble Alpes

Édition imprimée

Date de publication : 15 juin 2008

Pagination : 139-156

ISBN : 978-2-84310-125-0

ISSN : 0151-1874

Référence électronique

Pierre-Yves Buard et Carole Dornier, "Éditer un cahier de travail de Montesquieu », Recherches \&

Travaux [En ligne], 72 | 2008, mis en ligne le 15 décembre 2009, consulté le 03 février 2021. URL

http://journals.openedition.org/recherchestravaux/100 ; DOI : https://doi.org/10.4000/

recherchestravaux.100 


\section{Éditer un cahier de travail de Montesquieu : les apports du numérique et de la TEI}

Le projet d'une édition électronique des manuscrits de Montesquieu est né des exigences et contraintes de l'édition papier, associées aux possibilités offertes par la numérisation de manuscrits ${ }^{1}$. La préparation d'une transcription de manuscrits requiert un accès fréquent à des documents fragiles, abîmés, parfois difficilement lisibles. La numérisation, a fortiori dans des versions offrant une image dans un format de haute définition, et l'utilisation de logiciels perfectionnés de traitement d'images offrent des ressources nouvelles aux chercheurs qui exploitent ces sources délicates à manier et parfois à interpréter. Dans le cas des manuscrits de Montesquieu, la numérisation, envisagée d'abord pour aider à l'édition papier, mais en concertation avec les institutions détentrices des fonds, permet leur mise à disposition d'un public élargi, associant la commodité du chercheur, le souci de valorisation du dépositaire des fonds, et l'intérêt croissant pour le patrimoine écrit. Cependant, les images numérisées de manuscrits demeurent difficiles à exploiter pour des non-spécialistes. Le statut des diverses inscriptions qui figurent sur l'espace de la page nécessite une interprétation, qui est l'objet d'un travail éditorial scientifique. Une véritable valorisation de ces sources numérisées passe par une transformation de l'objet matériel en texte, c'est-à-dire en contenu signifiant. La mise en ligne de la transcription offre non seulement la possibilité d'interpréter l'image; elle permet également de développer des outils de

I. En complément de cet article, un diaporama a été mis en ligne sur le site de la MSHAlpes : http://www.msh-alpes.prd.fr/Actualites/Manuscrits.htm. 
recherche pour extraire des données et avancer dans la confirmation de certaines hypothèses. Conçue à la fois comme un outil de recherche, une aide au travail de l'édition d'un manuscrit, et un instrument de valorisation d'un patrimoine intellectuel d'un intérêt exceptionnel, l'édition électronique des Pensées a été conçue à partir du choix d'une DTD préexistante, la TEI, particulièrement adaptée à un type de manuscrit qui n'est ni l'état préparatoire d'une œuvre, ni la source diplomatique éditée par les chartistes. La nature même du document implique un travail d'édition critique qui vise à fournir un texte intelligible directement utilisable par le lecteur, tout en y associant par un apparat critique et des notes d'éditeur une série d'informations éclairant le texte et son contexte. Au choix de la TEI pour l'édition en ligne, s'est ajouté celui du moteur de recherche PhiloLogic $\bigcirc \AA$, développé au sein du projet ARTFL (American Research on the Treasury of French Language, ARTFL Project) de l'Université de Chicago en collaboration avec le département Electronic Text Services de la bibliothèque de l'institution (The University of Chicago Library). Ce sont ces choix effectués en fonction de la nature particulière de ce manuscrit et des visées de cette édition qui sont ici présentés.

Le corpus des manuscrits de Montesquieu est d'un intérêt exceptionnel car la conservation des manuscrits à usage privé qui ont servi à l'élaboration d'une œuvre ne correspond pas aux pratiques du XVIII ${ }^{\mathrm{e}}$ siècle, même s'il faut relativiser la rareté de ces documents qui ne survivaient pas en général au "passage dans l'atelier d'imprimerie ${ }^{2} »$. Or ce sont des milliers de pages écrites par Montesquieu lui-même ou par ses secrétaires qui sont déposés à la Bibliothèque de Bordeaux, à la Bibliothèque nationale de France et dans quelques autres lieux comme la Bibliothèque Bodmer à Genève'. Montesquieu a laissé de nombreux manuscrits de travail, cahiers, recueils de notes, dossiers documentaires et états antérieurs aux imprimés, qui permettent de reconstituer une méthode de documentation et de réflexion. Le recueil des Pensées, qui est le premier manuscrit traité dans le projet d'édition électronique présenté ici, accompagne Montesquieu dans sa carrière d'écrivain pendant presque trente ans entre $1726-1727$ et 1754 . Ce recueil se distingue des brouillons d'auteur qui, aux XIX et $\mathrm{XX}^{\mathrm{e}}$ siècles, permettent de suivre le processus de création d'un écrivain à travers ses différentes étapes, grands corpus sur lesquels s'est d'abord développée la critique génétique,

2. Voir l'introduction de J.-L. Lebrave et A. Grésillon à Écrire aux XVII et XVIII siècles : genèses des textes littéraires et philosophiques, CNRS éditions, 2000, p. 7-I 5 .

3. Voir G. Benrekassa, «Le legs manuscrit de Montesquieu : des CEuvres de 1758 aux Euvres complètes du XXI" siècle» et P. Rétat, «Annexe I. Liste des manuscrits subsistants», Euvres complètes de Montesquieu. I [Lettres Persanes], Société Montesquieu, édition dirigée par J. Ehrard et C. Volpilhac-Auger, Oxford, Voltaire Foundation, 2004, p. XLIV-LXXIV. 
stimulée par l'abondance des matériaux qui font souvent défaut pour les époques antérieures. La notion même de création littéraire, associée au concept d'auteur, qui sous-tend ces études, n'est pas adaptée à des manuscrits comme le Spicilège et les Pensées. Montesquieu recueille dans une certaine mesure l'héritage humaniste dont il prolonge les méthodes de travail : annotations, extraits, recueils de citations ${ }^{4}$. Le manuscrit des Pensées se différencie également des manuscrits de mise au net, premiers états d'ouvrages qui ont été imprimés ou étaient destinés à l'impression, ou ouvrages écrits à la main pour une diffusion publique. L'auteur consigne dans ce recueil des réflexions qu'il laisse en attente pour une réutilisation ultérieure dans des ouvrages dont certains sont restés à l'état de projets. Parmi ces fragments, on trouve des citations, des propos entendus, des maximes ou remarques originales, des développements qui entraient dans un projet d'ouvrage, des chutes d'ouvrages publiés. De cette façon, on peut parler d'archivage de matériaux pour son œuvre. On voit apparaitre dans le recueil la façon dont Montesquieu conserve, jette, transforme, réutilise des idées et des formulations, dont il tire parti de ce qu'il a lu, entendu en société, proféré lui-même à des tiers, observé dans ses voyages, pour en nourrir ses hypothèses. On y aperçoit la façon dont il utilise des sources diverses mais aussi dont il fait jaillir sous sa plume la formule frappante et spirituelle, qui donne corps à une intuition, dont il retravaille des énoncés pour transformer, avec la phrase, l'idée mêmes.

\section{La délimitation des parties d'un texte fragmentaire}

Le caractère éclectique et fragmentaire du recueil pose un premier problème de structuration : celui de la délimitation des parties du texte, qui n'est pas organisé comme un texte destiné à la publication, en titres, chapitres, sections, etc.

La version numérique permet de rapprocher la transcription en mode texte du manuscrit en mode image en adoptant un même découpage par folio ou page. Dans une version papier, la pagination de l'édition impose la perte de

4. Voir à ce sujet G. Benrekassa, «La fabrique de la pensée : l'étude, la connaissance et l'usage du manuscrit dans l'Esprit des lois de Montesquieu», Ecrire aux XVIT et XVIIT siècles, op. cit., p. IO5I I 6; C. Volpilhac-Auger, «L'ombre d'une bibliothèque : les cahiers d'extraits de Montesquieu», Lire, écrire, copier, sous la direction de É. Décultot, CNRS éditions, 2003, p. 79-90.

5. Sur le statut et les spécificités du recueil, voir C. Dornier, «La mise en archive de la réflexion dans les Pensées de Montesquieu», Revue Montesquieu, nº 7, 2003-2004, p. 25-39; «Les Pensées de Montesquieu et la tradition des formes brèves", Poétique de la pensée. Mélanges offerts à Jean Dagen, textes réunis par B. Guion, S. Menant, M. Susana Seguin et Ph. Sellier, Champion, 2006, p. 363-377. 
l'organisation de l'original sur l'espace de la page. Il en résulte une difficulté, dans le manuscrit qui nous occupe, pour faire apparaitre à leur place et avec le statut particulier et incertain qui est le leur les annotations marginales, tantôt à considérer comme des notes d'auteur, tantôt plus proches d'additions. Les numéros des Pensées qui ont été assignés par commodité par Louis Desgraves, gardés aussi pour l'usage du lecteur dans l'édition en cours, peuvent être mis en retrait dans l'édition électronique, de façon à faire apparaître des continuités entre les fragments, là où la numérotation Desgraves sépare arbitrairement. Dans le passage qui suit, les paragraphes se succèdent autour d'un même thème ( $\mathrm{n}^{\text {os }}$ I 25 à I 27$)^{6}$. La numérotation Desgraves rompt la continuité sémantique et dénature ainsi l'original en créant une séparation artificielle. La TEI permet de prendre en compte le numéro de volume, la pagination et le foliotage du manuscrit, mais également la numérotation de l'éditeur, tout en ménageant la possibilité de placer le numéro à un endroit sur la page d'écran qui ne rompe pas la continuité apparaissant sur le manuscrit. Le balisage choisi permet la conservation d'une numérotation partagée par la communauté scientifique, et qui facilite la consultation par concordance avec les éditions papier précédentes. Il offre aussi la possibilité de respecter une caractéristique du manuscrit : la relative indécision des limites de parties de texte, dans la mesure où la numérotation peut ne pas apparaitre ou être placée à un endroit qui ne perturbe pas la continuité du texte.

Exemple :

La transcription selon les normes de l'édition papier est suivie de la version XML balisée et du résultat à l'écran :

\section{Transcription :}

\section{[125]}

Je ne sçais pas si les anciens avoient de meilleurs esprits, mais par le changement des tems il est arrivé que nous avons $<\mathrm{de}>\uparrow$ quelquefois de +7 meilleurs ouvrages,

$$
\text { [1 26] }
$$

Mais pour juger des beautés d'Homere il faut se mettre dans [p. II 8] le $<$ camp des> camp des Grecs non pas dans une armée françoise.

6. Les Pensées sont citées en utilisant la numérotation de l'édition Desgraves (Robert Laffont, «Bouquins», I99I) mais selon le texte transcrit pour l'édition en cours à la Voltaire Foundation.

7. Addition autographe. 
Nous pouvons aimer a voir la representation des moeurs d'un peuple barbare pourvü qu'on y trouve les passions qui plaisent et qui remuent $<$ et quoique les heros n'ayent pas attrapé comme nous la maniere la plus destructive du genre humain, cela n'y fait rien, il suffit que leurs passions nous touchent>, nous aimons a voir les mêmes passions sur un fonds nouveau, nous aimons bien mieux entendre le vizir Acomat parler de sa maniere d'aimer que $<$ le fade $>$ Bajazet naturalisé françois.

\section{Balisage XML correspondant :}

$<$ divi id=pn" 125 ">

$<\mathrm{p}>$ Je ne sçais pas si les anciens avoient de meilleurs esprits, mais par le changement des tems il est arrivé que nous avons

$<$ del $>$ de $</$ del $><$ add hand $="$ M"

place $=$ "au dessus de la ligne" $>$ quelquefois $\mathrm{de}</$ add $>$ meilleurs ouvrages. $</ \mathrm{p}>$

$</ \operatorname{div}$ i $>$

$<$ divi id="pni 26 " $>$

$<\mathrm{p}><$ note type $=$ "thème" $><$ add hand $=" \mathrm{~T} "$

place $=$ "en marge" $>$ Homere $</$ add $></$ note $>$ Mais pour juger des beautés

d'Homere il faut se mettre dans $<$ pb n="pi i 8 " $/>$ le $<$ del $>$ camp

des $</$ del $>$ camp des Grecs non pas dans une armée françoise. $</ \mathrm{p}>$

$</ \operatorname{divi}>$

$<$ divi id="pni $27 ">$

$<$ p $><$ note type $=$ "thème" $><$ add hand="T"

place $=$ "en marge" $>$ Passion $</$ add $></$ note $>$ Nous pouvons aimer a voir la representation des moeurs d'un peuple barbare pourvü qu'on y trouve les passions qui plaisent et qui remuent $<$ del $>$ et quoique les heros n'ayent pas attrapé comme nous la maniere la plus destructive du genre humain, cela n'y fait rien, il suffit que leurs passions nous touchent $</$ del $>$, nous aimons a voir les mêmes passions sur un fonds nouveau, nous aimons bien mieux entendre le vizir Acomat parler de sa maniere d'aimer que $<$ del $>$ le fade $</$ del $>$ Bajazet naturalisé françois. $</ \mathrm{p}>$

$</ \operatorname{div}$ I $>$ 


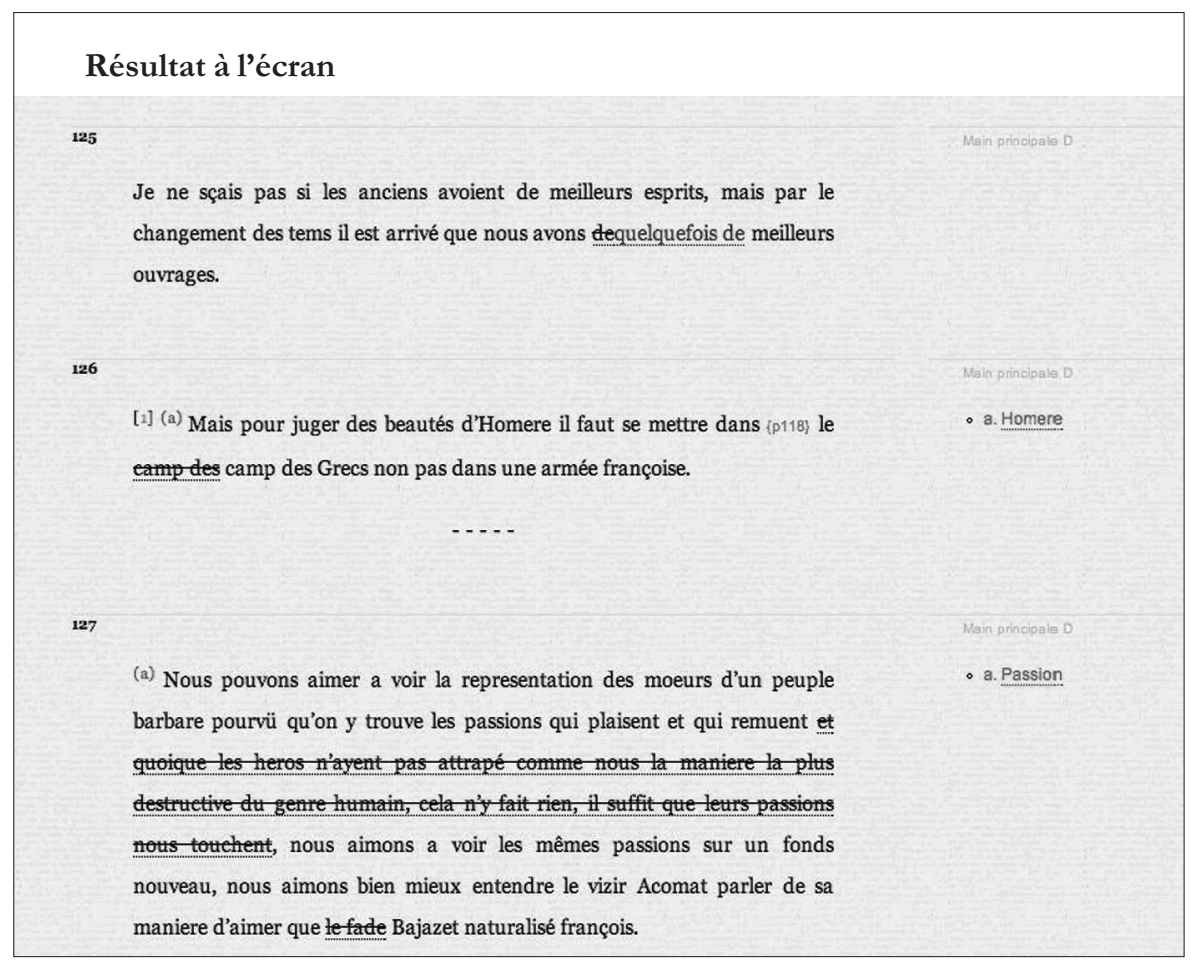

La balise < divi > associée à l'attribut «id» permet de définir le contenu du fragment numéroté (<divi id="pni 25 " $>$ ). La numérotation correspond dans cet exemple à la division en paragraphe (balise $<\mathrm{p}>$ ).

\section{Les Pensées, texte stabilisé}

Le processus que l'on peut repérer et étudier dans les Pensées est un état intermédiaire entre la documentation et la création. L'édition du manuscrit participe donc dans une certaine mesure des études génétiques parce qu’il donne à voir le processus de documentation, de travail sur le style, de transformation d'énoncés utilisés parfois dans les ouvrages qui ont été imprimés. Des différences notables distinguent cependant ce manuscrit des corpus considérés comme plus familiers aux études génétiques ${ }^{8}$. Le document qui

8. Sur les pratiques de composition et le statut d'auteur, qui éclairent les différences de production textuelle et engagent différentes approches relevant de la "génétique», voir J.-L. Lebrave et A. Grésillon, op. cit., p. 7- I 5 ; F. Moureau, «Du bon usage des manuscrits et des autographes littéraires : le cas du XVIII siècle», [Travaux de littérature XI.] Le Manuscrit littéraire : son statut, son histoire, du Moyen Áge à nos jours, numéro coordonné par L. Fraisse, Strasbourg, Publications de l'ADIREL, I998, p. I95-2 I I. 
nous intéresse ne témoigne ni de la genèse d'un ouvrage précis, ni des versions successives d'un texte en cours d'élaboration. Il ne s'agit pas d'un dossier génétique présentant plusieurs couches de texte à analyser pour restituer le travail d'écriture. Ce cahier de travail n'est pas destiné à la publication et il ne constitue pas une étape de textualisation en vue d'un texte définitif. C'est un texte de nature privée mais stabilisé pour des raisons utilitaires. Il s'adresse à Montesquieu lui-même qui le relit pour y puiser des énoncés ou ensembles d'énoncés en vue d'utilisations exogènes. La lisibilité du texte s'impose pour pouvoir mener à bien ce travail de réutilisation. La notion de texte stabilisé ou texte idéal conditionne les choix de transcription et les choix éditoriaux.

Dans le cas de ce cahier de travail, cela revient à restituer sous la forme d'un texte imprimé tous les éléments du manuscrit conservés pour être relus, c'est-à-dire tout ce qui, de la main de l'auteur ou d'un scripteur dont on peut savoir qu'il a travaillé sous sa direction, n'est pas biffé, ce qui implique de prendre en compte l'ensemble des additions placées entre les lignes ou en marge. Les éléments biffés sont alors rejetés en dehors du texte pour constituer l'apparat critique, qui informe le lecteur des versions antérieures sous la forme du texte biffé rétabli ou du signalement des additions. Ce qui est produit dans cet apparat critique, ce ne sont ni les variantes collationnées à partir des différents états imprimés, ou témoins utiles dans le cas des chartes ${ }^{9}$, ni les réécritures des dossiers génétiques qui récusent la référence à un texte modèle ${ }^{10}$. L'apparat critique est donc constitué par des informations que l'on extrait de l'examen de la page manuscrite pour faire ressortir en l'analysant ce qui est voulu comme définitif et en plaçant séparément ce qui témoigne d'une intention antérieure. La transcription préparée pour l'édition papier utilise, conformément aux directives générales données par le conseil scientifique de la Société Montesquieu ${ }^{11}$, responsable de l'édition des CEuvres complètes en cours, des sigles conventionnels. On aboutit à une transcription linéarisée qui ne restitue pas les données topographiques de l'original. Les choix de transcription vont à la fois faire perdre et ajouter de l'information, par rapport au contenu de l'image numérique ${ }^{\mathrm{I} 2}$.

9. Sur cette notion de témoin et sur l'édition des chartes avec balisage TEI, voir «L'édition critique des sources historiques : du support papier au Web», http://www.lespetitescases.net, p. 3 .

I . A. Grésillon, Éléments de critique génétique, PUF, I994, p. 75-76.

I I. http://montesquieu.ens-lsh.fr.

I 2. Sur la dualité texte/image dans la gestion des manuscrits comme documents numériques, voir A. Crasson et J.-D. Fekete, «Structuration des manuscrits : du corpus à la région», Proceedings of CIFED 2004, La Rochelle, [en ligne] www.lri/-fekete/ps/crassonfeketecifedo4final.pdf, p. I62-I 68 . 
En effet, le résultat attendu pour l'édition papier implique que l'information contenue sur la page manuscrite soit analysée et présentée en séparant la page de l'imprimé final en trois zones de textes, qui correspondent à trois fichiers séparés fournis à l'éditeur: un fichier texte, un fichier apparat critique et un fichier «notes d'auteur» sur lequel nous reviendrons. Nous présentons dans l'exemple suivant les étapes de préparation de la version papier que nous confronterons ensuite à la version électronique encodée selon les recommandations de la TEI.

Exemple :

I. Page du manuscrit en mode image :

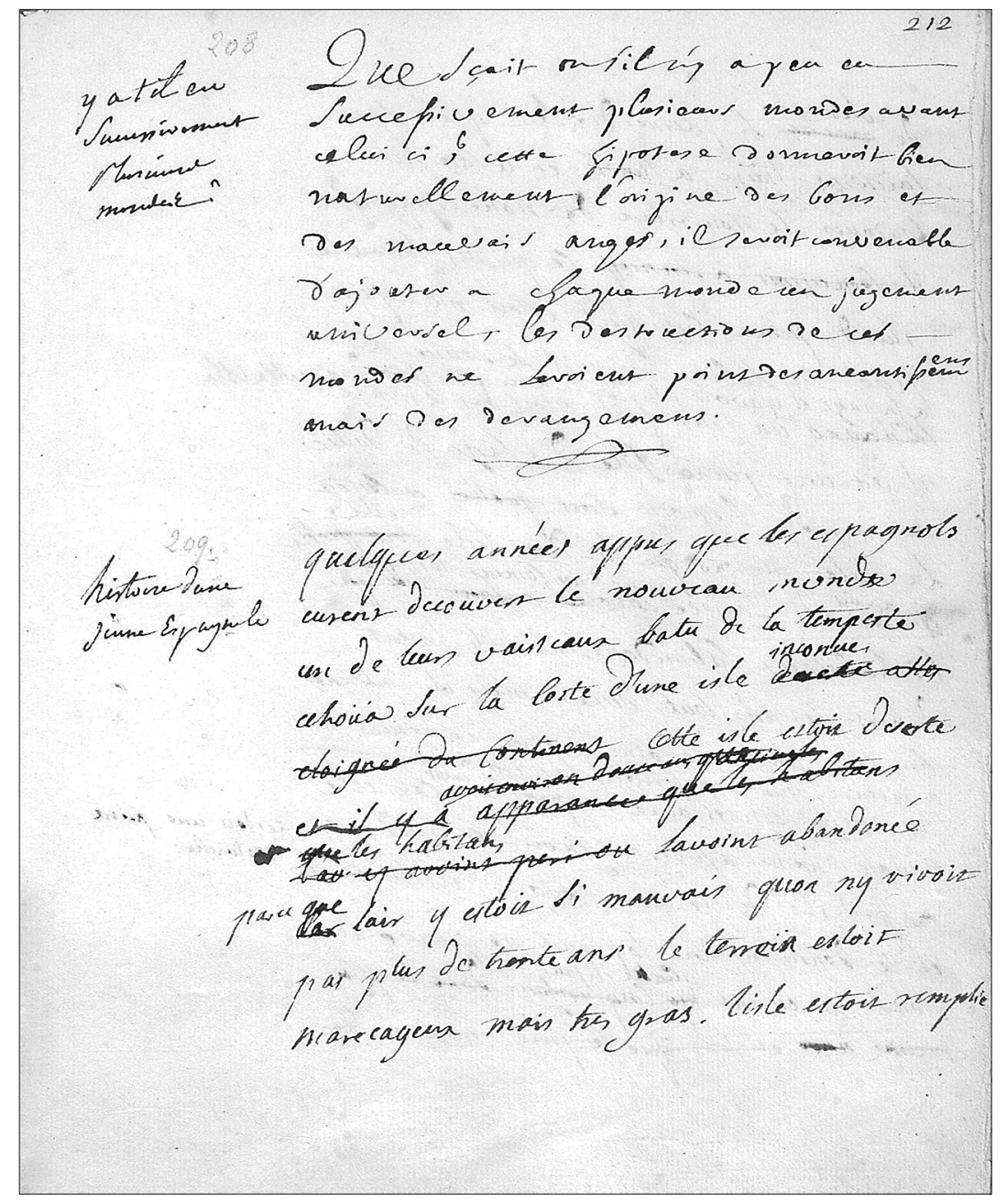


2. Première étape de la transcription sans séparation analytique

$$
\text { [n } \left.\mathrm{n}^{\circ} 209\right]
$$

Quelques années appres que les Espagnols eurent decouvert le nouveau monde un de leurs vaisseaux batu de la tempeste echoüa sur la coste d'une isle $<$ deserte asses eloignée du continent $>\uparrow$ inconue + cette isle estoit deserte $<$ et il y a apparance que les habitans $<$ l'av $>\uparrow$ avoit environ douze ans que [mot illisible les $+<\mathrm{y}$ avoient peri ou $>\uparrow<$ et que $>$ les habitans + l'avoint abandonée $<$ car $>\uparrow$ parce que + l'air y estoit si mauvais qu'on n'y vivoit pas plus de trente ans le terrein estoit marecageux mais tres gras. L'isle estoit remplie [p. 21 3] de $<$ vaches $>\uparrow$ chevres + si pleines de lait qu'elles se laissoint traire a l'envi et ce lait fut toujours la nourriture de notre Espagnol <il comencoit a s'ennuy > ce qui lui faisoit le plus de peine c'est qu'il estoit nu ayant jetté ses habits lors qu'il se sauva $<a$ la nage $>\uparrow a$ la nage il y avoit plus de six mois qu'il estoit dans cette isle lors + $\mathrm{q}[\mathrm{u}$ '] un jour qu'il estoit sur le rivage il vit une jeune fille de l'age de douze ans qui s'y beignoit < soit qu'elle $>$ et c'estoit la seule persone qui fut dans l'isle $<$ soit qu'elle y eut $>$ elle avoit + esté laissée <encore enfant lors que $>\uparrow j$ je ne scai coment lors que+ les habitans l'abandonerent, ils furent d'abort surpris touts deux mais ils sentirent bientost qu'ils n'estoint point ennemis $\uparrow$ a mesure que l'Espagnol s'approchoit+ la jeune Americaine s'approcha $\uparrow$ aussi+, car elle n'avoit point apris a ignorer ce qu'il est impossible de ne pas scavoir [209/mI] ils s'aimerent et se donnerent une foy qu'ils ne pouvoient pas violer ils eurent $<$ qu $><$ cinq enfans dont le plus vieux n'av $><$ et lors que $>\uparrow$ quatre enfans + le pere mourut $<$ l'ai $>$ [p. 2I 4 [ \{ l'ainé n'avoit que $<$ cinq ans $>\uparrow$ de quatre a cinq ans $+>$ et la mere ne survecut que de quelques $<$ mois pendant lesquels elle apprit $><$ les chevres acoutumées $>\uparrow$ jours laissant un petit peuple dont le plus agé n'avoit que trois ans et demi les chevres acoutumées + a venir $<$ presenter $>$ alaiter les petits enfans continuerent toujours $<$ il y a apparance que l'aisné mourut et il se leva $>$ ils crurent $<$ et la mere ne lui survecut qu et arriverent $>$ sans $<$ accident a l'age de $>$ raison $>$ \}

$[209 / \mathrm{mI}]$ C'estoit une priere naturelle

Dans cette première étape on constate déjà la difficulté à transcrire une portion de texte en marge «C'estoit une priere naturelle», dont le statut est équivoque : note ou addition? Cette portion de texte est ici traitée comme une note d'auteur, avec un appel de note, que le transcripteur doit placer avec un certain arbitraire ([209/mI]) et le texte de la note mis de côté en vue de son insertion dans un fichier séparé. 
3. Deuxième étape de la transcription avec séparation en trois fichiers :

\section{Texte de base :}

$$
[209]^{13}
$$

Quelques années appres que les Espagnols eurent decouvert le nouveau monde un de leurs vaisseaux batu de la tempeste echoüa sur la coste d'une [29] isle inconue. Cette isle estoit deserte les habitants l'avoient abandonée parce que[x 29] l'air y estoit si mauvais qu'on n'y vivoit pas plus de trente ans le terreïn estoit marecageux mais tres gras. L'isle estoit remplie [p. 2 I 3] [30]de chevres $\left[\mathrm{x}_{3} \mathrm{O}\right]$ si pleines de lait qu'elles se laissoint traire a l'envi et ce lait fut toujours la nourriture de notre [3 $\mathrm{I}$ ] Espagnol ce qui lui faisoit le plus de peine c'est qu'il estoit nu ayant jetté ses habits lors qu'il se sauva a la nage il y avoit plus de six mois qu'il estoit dans cette isle lors q[u']un jour qu'il estoit sur le rivage il vit une jeune fille de l'age de douze ans qui s'y beignoit et c'estoit la seule persone qui fut dans l'isle elle avoit esté laissée je ne scai coment lors que les habitans l'abandonerent, ils furent d'abort surpris touts deux mais ils sentirent bientost qu'ils n'estoint point ennemis a mesure que l'Espagnol s'approchoit la jeune Americaine s'approcha aussi, car elle n'avoit point apris a ignorer ce qu'il est impossible de ne pas scavoir [209/ $\mathrm{mI}$ ] ils s'aimerent et se donnerent une foy qu'ils ne pouvoient pas violer ils eurent quatre enfans le pere mourut [p. 2I 4 ] et la mere ne survecut que de quelques jours laissant un petit peuple dont le plus agé n'avoit que trois ans et demi les chevres acoutumées a venir alaiter les petits enfans continuerent toujours[3 I ]

\section{Apparat critique}

[29] isle $<$ deserte asses eloignée du continent $>\uparrow$ inconue + cette isle estoit deserte $<$ et il y a apparance que les habitans $<$ l'av $>\uparrow$ avoit environ douze ans que [mot illisible] les $+<$ y avoient peri ou $>\uparrow<$ et que $>$ les habitans + l'avoint abandonée $<$ car $>\uparrow$ parce que +

$[30]$ de $<$ vaches $>$ chevres +

[3 I] Espagnol <il comencoit a s'ennuy $>$ ce [...] sauva $<$ a la nage $>$ a la nage il y avoit plus de six mois qu'il estoit dans cette isle lors $+\mathrm{q}[\mathrm{u}]$ un $[\ldots]$ beignoit $<$ soit qu'elle $>$ et $[\ldots]$ l'isle $<$ soit qu'elle y eut $>$ elle avoit + esté laissée $<$ encore enfant lors que $>$ je ne scai coment lors que + les $[\ldots]$ ennemis $\neq a$ mesure que l'Espagnol s'approchoit + la [...] s'approcha aussi,$+ \operatorname{car}[\ldots]$ eurent $<$ qu $><$ cinq enfans dont le plus vieux n'av $><$ et lors que $>$ quatre enfans + le pere mourut $<$ l'ai $>$ [p. ${ }_{21}$ ] $]<$ l'ainé n'avoit que $<$ cinq ans $>$ de quatre a cinq ans $+>$ et la $[\ldots]$ quelques $<$ mois pendant lesquels elle apprit $><$ les chevres acoutumées $>$ jours laissant un petit peuple dont le plus agé n'avoit que trois ans et demi les chevres

I 3. En marge : «Histoire d'une jeune Espagnole». 
acoutumées + a venir <presenter $>$ alaiter $[. .$.$] toujours <$ il y a apparance que l'aisné mourut et il se leva $>$

\section{Notes de Montesquieu}

$[209 / \mathrm{mi}]$ C'estoit une priere naturelle

La transcription permet la lisibilité et l'analyse de ce qui a été voulu comme texte définitif et qui est ainsi extrait pour être placé dans un fichier texte, qui constituera l'élément central de la page éditée en version papier. La perte se situe du côté du statut exact des portions de texte placées dans les marges. On a vu les difficultés de la séparation de la note marginale et de son signalement par un appel dont le placement est laissé à l'appréciation du transcripteur. On voit aussi qu'un titre ou résumé donné en marge, sur lequel nous reviendrons, n’a pu être pris en compte que par une note d'éditeur : «En marge : "Histoire d'une jeune Espagnole"». La lecture de l'apparat critique est par ailleurs rendue difficile par le nombre important de portions biffées ou ajoutées.

Le choix des balises de la TEI permet d'éclairer la spécificité du manuscrit et les caractéristiques de l'apparat critique. L'utilisation des deux balises $<$ add $>$ et $<$ del $>$ suffit à rendre compte de ce que nous apprend la transcription. Un texte stable est produit par addition, par intercalation de lettre, mot, phrase, et par suppression de lettre, mot, phrase, paragraphe rayé par l'auteur ou celui qui travaille sous sa direction. La recherche des balises adéquates a permis de préciser rigoureusement la différence entre un apparat critique d'imprimé présentant plusieurs états du texte et l'apparat critique de ce type de manuscrit, alors que la présentation de l'édition papier tend à supprimer ces différences. Ainsi c'est moins le chapitre i 2 (Critical apparatus) des directives de la TEI qui nous a fourni les balises correspondant à nos besoins ${ }^{14}$ que le chapitre I I (Representation of Primary Sources ${ }^{\mathrm{I}}$ ). Les notions de «témoin» (witness $<$ wit $>$ ) et de lectures (reading $\langle\mathrm{rdg}\rangle$ ) ne sont pas opérantes ici puisque nous n'avons pas affaire à une comparaison de différents états d'un même texte. L'utilisation d'une structure $<$ app $>$ (apparat critique) dans les balises $<$ add $>$ et $<$ del $>$ peut être une façon de faire apparaitre la substitution d'un texte à un autre. On choisit aussi la balise < unclear $>$ pour rendre compte des portions de texte illisibles qui ont résisté à l'effort du transcripteur. La balise

I4. TEI Guidelines, «Critical Apparatus», http://tei.oucs.ox.ac.uk/P5/Guidelines-web/en/ $\mathrm{html} / \mathrm{TC} . \mathrm{html}$.

I 5. «Representation of Primary Sources», http://www.tei-c.org/release/doc/tei-p5-doc/en/html/ PH.html\#body.I_div.I I_div.s. 
$<$ sic $>$ permet de souligner une graphie aberrante tout en la conservant et en la signalant.

Ces balises permettent de traiter un texte unique stabilisé avec un seul état antérieur biffé et une seule addition/correction. Dans la plupart des cas on a donc tout au plus deux états du texte : le texte stabilisé avec suppressions et additions; le texte antérieur apparu sous les biffures et par suppression des additions. La difficulté de lecture de l'apparat critique sur une version papier, mais aussi la dénaturation des spécificités de l'original, en particulier le statut incertain des notes marginales (addition ou explication de l'auteur), sont des problèmes en partie résolus par l'édition en ligne avec ce type d'encodage. Le lien entre l'image et le texte permet de repérer l'espace occupé sur la page manuscrite par telle portion de texte: on utilise la balise $<\mathrm{pb}>$ (page break) enrichie par l'attribut «n» contenant le numéro de la nouvelle page (voir paragraphe numéro i 26 de l'exemple p. I 42). Pour encoder la note marginale, on utilise les balises <note>, <add> et l'attribut «place» qui indique la localisation sur la page. Le lecteur peut choisir de visualiser le texte stabilisé, débarrassé des signalements d'additions ou de suppressions. Il peut aussi visualiser le texte transcrit avec ses additions et ses suppressions : le texte supprimé apparait en caractères barrés et le texte ajouté apparait en couleur, les informations sur les ajouts (scripteur et position) sont gérées par un système de $p \circ p-u p$.

\section{Identification des scripteurs et chronologie}

Une des caractéristiques du manuscrit en question tient aussi à la variété des mains qui sont intervenues. Comme dans d'autres manuscrits de Montesquieu, en particulier son autre recueil de travail, le Spicilège ${ }^{16}$, l'auteur a employé successivement plusieurs secrétaires. Si nous avions affaire à un manuscrit autographe, ses enseignements sur la chronologie de la réflexion et du travail de Montesquieu seraient réduits et il nous faudrait nous contenter des dates et mentions permettant de contextualiser certains fragments. Les problèmes de vue de Montesquieu et sa façon de travailler en faisant recopier à des secrétaires des notes prises sur des feuilles volantes nous offrent, comme dans le cas du Spicilège, mais plus encore, la possibilité de fixer des limites chronologiques à des séquences contenues dans le manuscrit des Pensées. Comme l'a déjà souligné Robert Shackleton, de longues suites de pages écrites par une main majoritaire, en alternance souvent avec Montesquieu, sont datables dans la mesure où l'on

I6. Euvres complètes de Montesquieu. I3, Spicilège, édité par R. Minuti et annoté par S. Rotta, Oxford, Voltaire Foundation, 2002. 
peut avoir une connaissance approximative des limites de la période au cours de laquelle un secrétaire s'est trouvé au service de Montesquieu. En effet, lorsqu'une écriture est identifiée, sa présence dans la correspondance ou dans d'autres écrits auxquels on peut assigner une date, permet de fixer un terminus a quo et un terminus ante quem pour l'intervention du secrétaire. Robert Shackleton, Georges Benrekassa et Catherine Volpilhac-Auger ont avancé, à quelques divergences près, des hypothèses pour cette chronologie qui concerne particulièrement les Pensées ${ }^{17}$. Ces investigations à partir des écritures peuvent être complétées par une approche codicologique, comme celle effectuée par Claire Bustarret sur certains manuscrits de Montesquieu, en utilisant la base de données MUSE (Manuscrits, Usages des Supports et de l'Écriture) ${ }^{18}$.

La datation des fragments qui composent le recueil est complexifiée par la façon dont Montesquieu a utilisé ces cahiers reliés. On constate en effet à l'examen des trois volumes que, dans les espaces restés vierges après une première transcription de fragments, s'intercalent des morceaux d'écritures postérieures. La datation des écritures confrontée à d'autres indices restitue ainsi une chronologie des remarques qui ne suit pas la progression linéaire de l'écriture d'une page à l'autre, du haut au bas de la page, et de gauche à droite sur la ligne. De nombreuses additions, de dimension variable, viennent s'intercaler entre les fragments, entre les lignes, dans les marges.

Le balisage selon les directives de la TEI permet d'encoder cette identification des différents scripteurs. Grâce à la balise $<$ hand $>$ pour l'identification du scripteur, à l'utilisation d'un code de couleurs, le lecteur peut immédiatement identifier et dater tout élément de la transcription, ce qui facilite grandement le travail de l'annotation. Ces informations sont capitales pour savoir à quel moment Montesquieu écrit ou fait écrire telle idée, mais également pour comprendre comment il revient sur un texte initial pour ajouter des explications et exemples, voire rectifier une idée, à la lumière de nouvelles connaissances et expériences, en particulier celles acquises pendant son périple en Europe de 1728 à $173 \mathrm{I}$.

I7. R. Shackleton, «Les secrétaires de Montesquieu», Montesquieu, Euvres complètes, Nagel, I950, t. II, p. xxxv-xliii; G. Benrekassa, Les Manuscrits de Montesquieu, Secrétaires, Écritures, Datations, Cabiers Montesquieu, no 8, 2004; Rolando Minuti, dans l'édition citée du Spicilège, p. 37 77 ; C. Volpilhac-Auger, «De la main à la plume. Montesquieu et ses secrétaires : une mise au point», Montesquieu en 2005, Studies on Voltaire and the eighteenth century, 2005, p. I03-I 5 I.

I 8. Cl. Bustarret, «Mobilité des supports, dynamique de l'écriture : l'apport des indices matériels", in Montesquieu, Euvre ouverte? (I748-1755), actes du colloque de Bordeaux (6-8 décembre 200I) présentés et publiés par C. Larrère, Cahiers Montesquieu, n 9, 2005, p. 229-252; "Le manuscrit 2506 : inventaire des papiers", L'Atelier de Montesquieu : manuscrits inédits de La Brède, Cabiers Montesquieu, éd. C. Volpilhac-Auger, nº 7, 2001, p. 284-305; «Étude des différents types de papier utilisés dans les Cahiers de correction des Lettres persanes (n.a.fr. I4365)", CEuvres complètes de Montesquieu. I, op. cit., p. 62 I-624. 
Si l'on revient à l'exemple précédent, présenté sous la forme des étapes de travail d'une édition papier, on peut donc voir à quels résultats aboutissent le balisage et la mise en ligne :

Exemple :

I. Arrière-plan du balisage TEI du no 209

$<\operatorname{divi}$ id="pn209">

$<$ p $><$ note type $=$ "thème" $><$ add hand="T" place $=$ "en marge" $>$ Histoire d'une jeune Espagnole $</$ add $></$ note $><$ handShift new="M" next="M"

old="D" / $>$ Quelques années appres que les Espagnols eurent decouvert le nouveau monde un de leurs vaisseaux batu de la tempeste echoüa sur la coste d'une isle $<$ del $>$ deserte asses eloignée du

continent $</$ del $><$ add hand $=" M "$ place $="$ au dessus de la ligne" $>$ inconue $</$ add $>$ cette isle estoit deserte $<$ del $>$ et il y a apparance que les habitans $</$ del $><$ del $>$ l'av $</$ del $><$ add hand="M"

place $=$ "au dessus de la ligne" $><$ del $>$ avoit environ douze ans que $<$ unclear $>$ [mot illisible $]</$ unclear $>$ les $</$ del $><$ /add $><$ del $>$ y avoient peri $</$ del $><$ add hand $=" M "$ place $="$ au dessus de la ligne" $><$ del $>$ et que $</$ del $>$ les habitants $</$ add $>$ l'avoint abandonée $<$ del $>$ car $</$ del $><$ add hand $=" \mathrm{M}$ " place $=$ "au dessus de la ligne" $>$ parce que $</$ add $>$ l'air $y$ estoit si mauvais qu'on n'y vivoit pas plus de trente ans le terrein estoit marecageux mais tres gras. L'isle estoit remplie $<\mathrm{pb}$ $\mathrm{n}=$ "p2 I 3 " $/>$ de $<$ del $>$ vaches $</$ del $><$ add hand="M" place $=$ "au dessus de la ligne" $>$ chevres $</$ add $>$ si pleines de lait qu'elles se laissoint traire a l'envi et ce lait fut toujours la nourriture de notre Espagnol $<$ del $>$ il comencoit a s'ennuy $</$ del $>$ ce qui lui faisoit le plus de peine c'est qu'il estoit nu ayant jetté ses habits lors qu'il se sauva $<$ del $>$ a la nage $</$ del $><$ add hand="M" place $=$ "au dessus de la ligne" $>$ a la nage il y avoit plus de six mois qu'il estoit dans cette isle lors $</$ add $>\mathrm{q}<$ corr resp $=$ "transcripteur" $>\mathrm{u}^{\prime}</$ corr $>$ un jour $[\ldots]$ 
2. Texte sans biffure du no 209 apparaissant à l'écran :

209

(a) Quelques années appres que les Espagnols eurent decouvert le nouveau monde un de leurs vaisseaux batu de la tempeste echoüa sur la coste d'une isle inconue cette isle estoit deserte les habitants l'avoint abandonée parce que l'air y estoit si mauvais qu'on n'y vivoit pas plus de trente ans le terrein estoit marecageux mais tres gras. L'isle estoit remplie $\{2213\}$ de chevres si pleines de lait qu'elles se laissoint traire a l'envi et ce lait fut toujours la nourriture de notre Espagnol ce qui lui faisoit le plus de peine c'est qu'il estoit nu ayant jetté ses habits lors qu'il se sauva a la nage il y avoit plus de six mois qu'il estoit dans cette isle lors q[u']un jour qu'il estoit sur le rivage il

- a. Histoire d'une jeune Espagnole

- 1. C'estoit une priere naturelle

- 2. C'est ce que j'ay reconu par ce que j'ay pu apprendre du pais et par l'histoire d'un d'un navire perdu dans ce dempsa temps la contre une isle du Mexique dont on garde memoire au Mexique ou quelques uns des gens du vaisseau se refugierent dans une chaloupe on ne put depuis decouvrir cette isle.

3. Texte du no 209 enrichi par biffures et mentions d'additions :

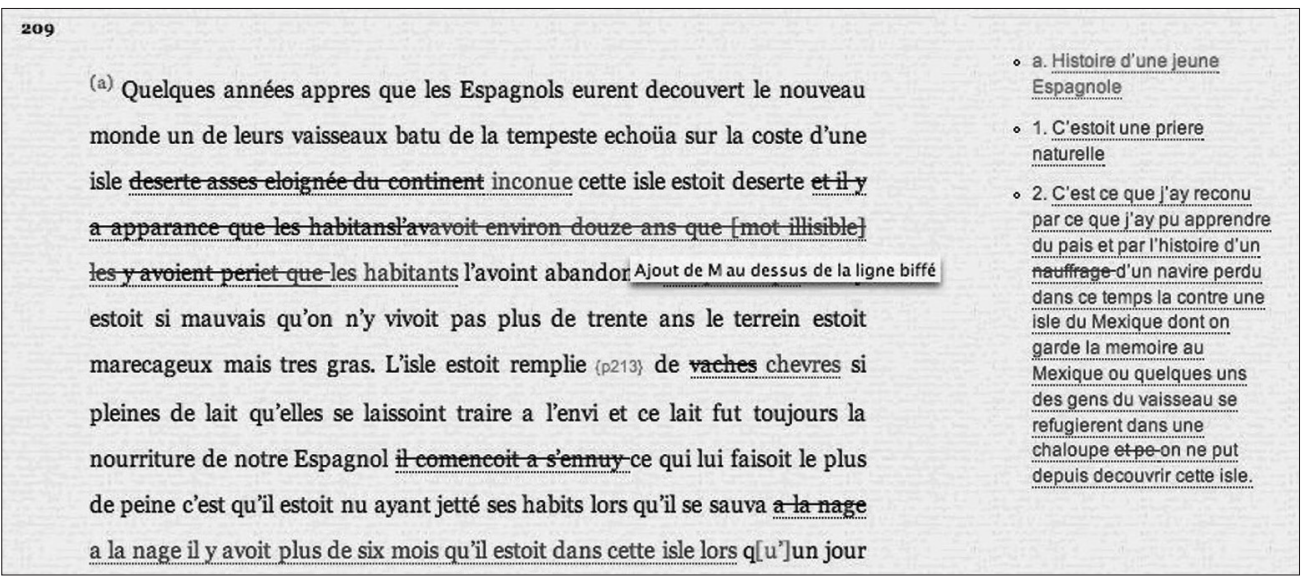

\section{Modes de navigation et extraction des données}

L'examen du manuscrit soulève encore d'autres interrogations : dans les marges, de façon régulière, la même écriture, non identifiée, court, consignant des expressions résumant un développement, des mots-clés, qui paraissent comme un moyen de désigner le contenu d'un fragment ou d'un paragraphe. Nous avons rencontré, dans le $\mathrm{n}^{\circ} 209$, cette écriture qui était traitée sous forme de note d'éditeur dans la transcription pour la version papier : «En marge : "Histoire d'une jeune Espagnole"». Cette écriture, négligée par tous les éditeurs et considérée comme sans autorité, transcrit une date à la fin du deuxième volume: 1749 . Sa disparition après 1750 , si l'on se fie à l'examen du troisième volume, corrobore l'hypothèse d'un travail effectué avant 1750, donc sous l'autorité de Montesquieu. Il ne s'agit pas d'un secrétaire de 
Montesquieu, mais d'un collaborateur chargé d'élaborer un mode de repérage - on dirait aujourd'hui de navigation - dans les manuscrits. Le balisage et l'édition en ligne permettent de rendre compte de la spécificité de cette écriture : elle est placée en marge sur la page mais n'a pas le statut de note marginale. Elle est encodée comme suit, si nous reprenons l'exemple du no 209 :

$<$ note type $=$ "thème" $><$ add hand="T" place="en marge" $>$ Histoire d'une jeune Espagnole $</$ add $></$ note $>$

L'encodage a permis d'extraire toutes les interventions de cette main et de faire apparaittre sa fonction : construire une table des matières permettant de naviguer dans un texte qui est un espace d'archivage. On trouve dans le recueil d'autres modes de navigation : renvois internes, notes concernant une utilisation prévue ou réalisée dans tel ou tel ouvrage. Après la publication des Considérations sur la grandeur des Romains et de P Esprit des lois, Montesquieu note ou fait noter en marge ceux des fragments employés. C'est ainsi que reviennent en marge de façon récurrente les mentions : «Mis dans les loix», «Mis dans les Romains».

Les recherches de termes simples, de phrases et de co-occurrences opérées au moyen du moteur de recherche PhiloLogic $\bigcirc \AA$, permettent d'extraire facilement tous les renvois à des extraits placés dans les ouvrages imprimés. On peut ainsi opérer des vérifications dans les imprimés mentionnés et faciliter le travail de l'annotation. PhiloLogic $\subset \circledR$ favorise des investigations sur des bases de données encodées : dans le cas qui nous occupe, ce moteur de recherche peut aider à la constitution d'index, en particulier à la recherche lexicale facilitant la constitution d'un index rerum. Il est particulièrement intéressant dans le cas d'un manuscrit à plusieurs scripteurs présentant beaucoup de variations morphologiques et orthographiques. Il permet également de faire apparaître le contexte des occurrences du mot et de faciliter ainsi dans l'annotation des renvois internes, de justifier le choix d'une entrée dans un index rerum en s'appuyant sur le nombre de ses occurrences, et celui d'un classement en sousentrées dans une entrée comportant beaucoup d'occurrences.

Exemple :

Recherche d'un nom propre, Caesar; résultats avec différentes orthographes :

3 correspondances, indiquées avec leurs fréquences dans la base complète.

Choisissez les mots à rechercher dans toute la base.

Rechercher ou Eflacer

$1 \Gamma$ caesar

2 Гcaesars

8 Гesar 
Recherche d'une expression récurrente pour confirmer et préciser une hypothèse :

«je disois»; calcul du nombre d'occurrences :

Recherche de je disois dans toute la base

II y a 117 occurrences correspondantes à votre recherche

Cette page contient les 25 premières occurrences. Reportez-vous au lien en bas de cette page pour voir le reste des occurences correspondante à votre recherche.

1. Pensées, volume $/[\mathrm{p} 260$ |Pensée 251]

dictum. II me semble que les Grecs estoint hardis pour le stile et timides pour la pensee M. dit qu'il est etone que les Anglois admirent tant les anciens puisqu'il $n$ 'y a persone qui les imite si peu et qui s'en ecarte davantage je disois a un Anglois qui me montroit quelque chose d'asses tendre coment 列 autheurs

2. Pensées, volume /[p304 | Pensée 275]

monoye d'un septieme les impots d'un dixieme suprimer la gabelle. - Grandes actions Je disols on ne scait coment faire pour faire une grande action notre interest s'y trouve on dit que c'est amour propre s'il ne s'y trouve pas on dit que c'est phanatisme. -

3. Pensées, volume /[ p338 | Pensée 339]

veut dire que vous souhaités de nous jetter dans les plus grands périls sans les partager. - Hongrie Je disois que je voulois voir la Honguerie parce touts les estats d'Europe avoint été come est la Honguerie a present, et que je voulois voir les moeurs de nos péres.

Le nom propre César peut être repéré dans l'ensemble des trois volumes quelle que soit son orthographe. La recherche de l'expression «je disois» permet de quantifier son usage et de confirmer qu'il s'agit d'une spécificité de ce recueil $^{19}$.

\section{Conclusion}

Par l'utilisation de la TEI sur un manuscrit de travail comme celui des Pensées de Montesquieu, on produit une transcription associée à la source en mode image, ce qui constitue à la fois une aide à la lecture et à l'interprétation. La mise en relation de l'image et du texte fait de la transcription un véritable accès au manuscrit, en augmentant sa lisibilité. Le balisage permet non seulement de restituer dans la transcription en ligne certaines spécificités du manuscrit, mais aussi de produire une analyse du document. Dans le cas présenté, la recherche des balises adéquates a permis de confirmer la différence entre un apparat critique d'imprimé présentant plusieurs états du texte et l'apparat critique de ce type de manuscrit, alors que la présentation de l'édition papier tend à supprimer ces différences. Elle établit également la distinction entre un dossier génétique et un cahier de travail comme celui qui est ici présenté.

Le choix du XML et de la TEI se justifie par la volonté de réaliser une édition facile de consultation, qui associe l'interprétation produite par la

I9. Voir C. Dornier, «La mise en archive... », art. cit., p. 33. 
transcription linéarisée avec la consultation de l'original en mode image. La possibilité de faire apparâttre ou de masquer une série de données facilite grandement l'exploitation du corpus. Par ailleurs la possibilité d'un encodage permettant d'extraire des données comme l'identité des scripteurs constitue une véritable aide à la recherche : en l'occurrence on progresse dans une vision synthétique de la chronologie des énoncés et des corrections pour aboutir à une vision diachronique de la documentation et de la réflexion de Montesquieu. En ajoutant à cette association texte/image un moteur permettant des recherches de termes et de phrases, on fait de l'édition électronique un instrument d'études linguistiques, stylistiques, une aide à l'annotation par la facilité à effectuer des renvois internes et à la constitution d'index. Images numériques et TEI constituent indéniablement des outils intéressants pour la valorisation et l'exploitation des corpus manuscrits, et particulièrement dans le cas présenté d'un manuscrit de travail dont ils permettent de restituer les spécificités. Loin de faire obstacle à une version papier dont la relative pérennité et la maniabilité demeurent des atouts, ils peuvent être conçus en complémentarité : les introductions et notes savantes étant réservées à la version papier, l'exploitation et la valorisation du manuscrit dans ses aspects matériels constituent l'intérêt de la version électronique, utilisée aussi pour renvoyer à l'objet-livre. 